\title{
REFLEXIÓN SOBRE LA ETNOMATEMÁTICA COMO POSIBILIDAD PEDAGÓGICA
}

\section{ARTÍCULO ORIGINAL}

SCHWANTES, Vilson ${ }^{1}$

XAVIER, Márcio Pizzete ${ }^{2}$

SCHWANTES, Eloísa Bernardete Finkler ${ }^{3}$

SCHWANTES, Daniel ${ }^{4}$

${ }^{1}$ Master en Ciencias de la Educación - Matemáticas, UNIJUI - RS. Licenciatura y Especialización en Ciencias y Matemáticas. Prof. Asistente del CCA - Centro de Ciencias Agrícolas, Campus del Mariscal Cândido Rondon, UNIOESTE, PR - Brasil.

2 Máster en Desarrollo Rural Sostenible-UNIOESTE, Especialista en Matemáticas, Física-UNIPAR, Gestión de Personas y Educación Especial con énfasis en Discapacidad Múltiple-UNIASSELVI, Graduado en Matemáticas con énfasis en FísicaUNIPAR.

3 Especialización en Enseñanza de Ciencias Exactas - Matemáticas, Física y Química. UNIOESTE - Universidad Estatal de Paraná Occidental. Graduación: Ciencias y Matemáticas. UNIJUI, RS. Profesor de la Escuela Estatal de Paraná.

${ }^{4}$ Profesor de Protección Fitosanitaria y Salud Humana en la Pontificia Universidad Católica de Chile, Departamento de Ciencias Vegetales, ocupa un puesto interdisciplinario compartido entre la Facultad de Agronomía e Ingeniería Forestal (FAIF), la Facultad de Medicina y la Facultad de Química. Profesor investigador asociado al Grupo de Estudio sobre Suelos y Medio Ambiente (GESOMA UNIOESTE). Máster en Agronomía por UNIOESTE, Doctorado en Agronomía por UNIOESTE (2013-2016) - Periodo Sandwich (Beca CAPES) de la Universidad de Lisboa, en el Instituto Superior de Agronomía (ULisboa). 
JUNIOR, Affonso Celso Gonçalves ${ }^{5}$

KRACKE, Elisa ${ }^{6}$

JUNIOR, Élio Conradi ${ }^{7}$

SCHWANTES, Vilson. Et al. Reflexión sobre Etnomatemática como posibilidad pedagógica. Revista científica multidisciplinaria base de conocimiento. año 04, Ed. 07, Vol. 11, págs. 148-165. Julio de 2019. ISSN: 2448-0959

\section{RESUMEN}

Este artículo reflexiona sobre la etnomatemática como una tendencia de la enseñanza que considera la naturaleza cultural del aprendizaje matemático, el contexto social donde se desarrolla este conocimiento, el papel del profesor en el contexto más amplio de las relaciones humanas asumiendo Investigador y mediador consciente de la práctica pedagógica. La investigación considera la importancia de la historia del conocimiento matemático, sus múltiples relaciones con otras áreas de especialización

5 Productividad de investigación Nivel $1 \mathrm{C}$ por CNPq en el área de Ciencias Ambientales con tres Postdocs, UEM-PR (Brasil), Universidad de Santiago de Compostela (España), UFG-GO (Brasil). Actualmente es profesor asociado en UNIOESTE-PR y actúa como profesor e investigador en el Centro de Ciencias Agrarias, enseñando química. Profesor del Programa de Maestría en Ciencias Agrícolas de la UEM. Actualmente es consultor ad hoc para CNPq, CAPES y Fundação Araucária. Actúa como consultor ambiental voluntario con MP-SP y CONAMA-DF.

${ }^{6}$ Licenciado en Agronomía - UNIOESTE - Universidad Estatal de Paraná Occidental - Licenciado en Administración - Unip - Universidade Pau

7 Estudiante de maestría en Agronomía (Producción de Plantas) de la Universidad Estatal de Paraná Occidental (UNIOESTE). Ingeniero Agrónomo egresado de UNIOESTE (2014-2018), actúa como investigador asociado al Grupo de Estudio de Suelos y Medio Ambiente (GESOMA - UNIOESTE). 
y su interrelación con las actividades diarias. El texto también reflexiona sobre las posibilidades y retos de la escuela para promover acciones pedagógicas que consideren las relaciones interpersonales en el proceso de (re) construcción del conocimiento. Llama a una investigación de investigación crítica de trabajo pedagógico, que de manera articulada busca establecer vínculos entre las prácticas cotidianas y las matemáticas escolares.

Palabras clave: etnomatemática, conocimiento social, habilidades matemáticas, actividades diarias, práctica pedagógica.

\section{INTRODUCCIÓN}

En el día a día del proceso pedagógico, los educadores continúan reflexionando sobre metodologías de enseñanza y formas de aprender. Uno de los principales desafíos de la escuela sigue contribuyendo a la formación de ciudadanos críticos, conscientes y activos en la sociedad. Una tarea compleja que requiere que los profesionales de la escuela y la educación vayan más allá del programa curricular, contenido y educación.

El mundo en el que vivimos, aunque no nos damos cuenta, siempre ha dependido fundamentalmente de las matemáticas. Siempre ha estado y está presente en prácticamente todo lo que nos rodea. Su aplicabilidad es relevante incluso en otras ciencias, como afirma D'ambrósio (1996, p. 31). "La tendencia de todas las ciencias es cada vez más matematizarem debido al desarrollo de modelos matemáticos que desarrollan fenómenos naturales de maneraapropiada."

Como vemos, las matemáticas se impregnan en todo el entorno social del ser humano y se evidencia en cada nuevo aprendizaje. Todas las profesiones actuales se apropian de los conocimientos matemáticos para crear, mantener y sostener reglas, fórmulas y conductas. El albañil, la costurera, el cocinero, el ingeniero, técnicos de todas las profesiones y profesionales de todos los sectores de la actividad humana dominan algunos conocimientos matemáticos, pero no siempre han aprendido los cálculos para resolver sus problemas en los bancos Escuela. 
Para Rosa Neto (2002), existe una relación significativa entre matemáticas y cultura, tanto como resultado de nuestra adaptación según nuestras necesidades de supervivencia a través de los tiempos, representando tanto a Científica.

Trabajar la Etnomatemática en el espacio escolar, según D'ambrósio (2008), es contribuir a las nuevas generaciones a conocer y reconocer una matemática mucho más cultural, vinculada a la vida cotidiana de varios grupos étnicos. Para D'ambrósio y Rosa (2016), se trata de una postura didáctica que busca una mejora en el proceso de enseñanza-aprendizaje de la disciplina con la incorporación al currículo matemático del conocimiento derivado de la vida del estudiante y de los valores humanos, como, por ejemplo, la cooperación, solidaridad y ética. Son conocimientos que promueven el aprecio y el respeto por las diferentes maneras que la humanidad utiliza diariamente para explicar, entender, entender y lidiar con las situaciones problemáticas que se enfrentan en la vida cotidiana.

Con esta conciencia sobre la importancia y el papel de las matemáticas en el desarrollo de los ciudadanos y la sociedad, este artículo, además de reflexionar, busca difundir etnomatemáticas, creyendo que como propuesta pedagógica puede contribuir en gran medida a Mitigar las dificultades de aprendizaje y la falta de interés de muchos estudiantes en el estudio de la disciplina.

En nuestra comprensión, un trabajo educativo centrado en esta perspectiva, hace que el estudiante se sienta más seguro de su propia capacidad para construir conocimiento matemático, en la búsqueda de soluciones a los problemas que encuentra dentro de su vida diaria. Para D'ambrósio (1996), sin embargo, es necesario que el maestro adopte una nueva postura en el aula, busque un nuevo paradigma que sustituya a la ya desgastada enseñanza-aprendizaje basado en una relación obsoleta de causa-efecto.

Pompeyo y Monteiro (2001, p. 14), advierten, enlatando que,

El cambio de paradigma, sin embargo, es un proceso complejo; Es necesario querer cambiar y creer que esto es posible. Más que descubrir 
que tenemos que cambiar, es necesario tener la convicción de que siempre hay una nueva forma de enseñar, que siempre es posible cambiar.

Los autores subrayan que trabajar pedagógicamente en esta perspectiva requiere que el profesor entienda y aclare el alcance de su papel, así como la importancia del contenido matemático con el que trabajan con los alumnos. En teoría, es necesario entender al estudiante de una manera integral, buscando identificar sus necesidades de desarrollo a nivel intelectual, físico, emocional, social y cultural. Por lo tanto, señalaron que,

Los profesores actuales se enfrentan a un gran reto: educados en un proceso fragmentado, tendrán que superar los límites que esta formación les impone y extrapolar los límites de los contenidos vistos de forma parcial e histórica (POMPEU y MONTEIRO, 2001, p. 15).

La tarea de la enseñanza y el aprendizaje, en este enfoque, es, de hecho, diferente de la tradicionalmente conocida. Es parte de lo que creemos que es consensuado, es decir, que todas las culturas y pueblos han desarrollado sus propias formas de explicar y modificar su propia realidad. Estos conocimientos y la creación de todos los procesos de desarrollo humano, propios de cada cultura, están en proceso, por supuesto, son dinámicos, en la fase de evolución y crecimiento constante, contribuyendo así en la elaboración del conocimiento sistado.

D'ambrósio (1993) enfatiza que las personas tienen una c[etno]ultura, tratan y explican su propia c[matema]ultura, sin embargo, cada una a su manera y forma[tica]. Este hallazgo dio lugar al nombre de Etnomathematics, que a su vez tiene en cuenta las explicaciones de las comunidades, goteándolas con las formas universales de conocimiento. Es un hecho que cada niño antes de llegar a la escuela sabe cómo lidiar perfectamente con su realidad, que está en constante evolución.

Según el autor, la Etnomatemática es parte del principio y la idea de incorporar los modelos vinculados a la tradición y cultura de los niños, reconociéndolos como válidos 
para el cumplimiento de sus propósitos. Así, la etnomatemáticamidse se mete con la curiosidad del niño, se vuelve primero, al entorno donde vive, cumpliendo una tarea que la escuela ha olvidado: liberar y desarrollar la creatividad de los estudiantes a través de proyectos Investigación.

Esta perspectiva pedagógica es la llamada etnomatemática, una tendencia $\mathrm{d}^{[8]}$ e la enseñanza de las matemáticas que considera en su práctica pedagógica los aspectos sociales y culturales de la humanidad. Se caracteriza por ser una tendencia docente que aporta a la discusión y busca reflexionar sobre las diversas formas de Matematizar presentes en nuestra realidad sociocultural.

Propone una educación crítica, cuya fundación está apoyada en los estudios y escritos de Ubiratan D'ambrósio, ${ }^{[9]}$ que apuntan a una propuesta metodológica de educación matemática que valora las experiencias de los estudiantes. Una visión con características que impregnan los procesos: antropológico, social y político, llevando a la discusión la cultura local de cada grupo social, como una actividad humana culturalmente determinada por el contexto donde se realiza.

Es en este aspecto que la etnomatemática, en las últimas décadas, se ha consagrado a nivel nacional e internacional como una propuesta didáctica-pedagógica para la enseñanza de la disciplina matemática. Según D'ambrósio (2001) "La etnomatemática es la matemática practicada por grupos culturales, como comunidades urbanas y rurales, grupos de trabajadores, clases profesionales, niños de cierto grupo de edad, sociedades indígenas, y tantos otros Grupos identificados por los objetivos y tradiciones comunes a los Grupos"(p. 9).

Es, según el autor, una tendencia de enseñanza donde el conocimiento se manifiesta de manera holística, es decir, permite a los diferentes grupos culturales conocer, entender y explicar su mundo, utilizando las matemáticas, desarrollando Capacidad de gestionar e interferir, a través de ella, en su realidad. Llama la atención la posibilidad de construir un currículo escolar que incorpore la vida sociocultural de los estudiantes en las prácticas pedagógicas, como una de las formas de inclusión social. 
En palabras de D'ambrósio (1993), "La etnomatemática es un programa que tiene como objetivo explicar los procesos de generación, organización y transmisión del conocimiento en diversos sistemas culturales y las fuerzas interactivas que actúan dentro y entre los tres Procesos" (p. 7).

En esta tendencia docente, el currículo es dinámico, contextualizado, reconoce la pluralidad cultural y social de los estudiantes, permite al estudiante convertirse en una asignatura activa, participativa en el proceso de transformación de su entorno local, regional y global.

En relación con el programa de Etnomathematics, D'ambrósio (2001, p. 17) también reflexiona sobre que,

El gran motivador del programa de investigación que yo llamo Etnomathematics es buscar entender el know-how matemático a lo largo de la historia de la humanidad, contextualizado en diferentes grupos de interés, comunidades, pueblos y naciones.

Pompeyo y Monteiro (2001), con respecto a la adopción de una práctica pedagógica que lleva el know/do al aula, argumenta que

Está impregnado de certezas que no son "dadas" por la ciencia, sino más bien por los valores, las tradiciones y el respeto por los que enseñan. Así, tiene una "certeza emocional" confirmada por la experiencia, diferente de la "certeza científica" que, en general, es susceptible a la demostración racional y a las matemáticas, aunque, cuando se enseña, también instituyen valores emocionales, presentes en la persona de Maestro (pág. 134 - 135).

En la acción pedagógica, la relación que se establecerá entre los procesos de enseñanza y aprendizaje es dialogante, porque es el contexto en estas interacciones lo que favorece las posibilidades de aprendizaje. En esta dinámica, llevar etnomathematics y conocer/hacer al aula significa recuperar las experiencias y 
conocimientos presentes en las actividades diarias de las diferentes clases trabajadoras, en un esfuerzo por superar el cienfismo del conocimiento Académico.

Sin embargo, Pompeyo y Monteiro (2001), en relación con el trabajo en el aula de los conocimientos formales e informales, señalaron que,

[...] Es necesario que un grupo conozca y reconozca los límites de su conocimiento para ir más allá y buscar lo nuevo con conciencia, conociendo y discutiendo la importancia y validez de este nuevo. Sólo así sabrá elegir cuándo y qué conocimiento usar (pág. 53-54).

En las escuelas en general, se enseña y aprende sólo las matemáticas dicta como oficial, mientras que en las actividades co[comercias, agrícolas, de prestação de serviços, entre outras]tidianas, aplican y utilizan conocimientos matemáticos no reconocidos oficialmente, pero construidos socialmente, validados y Valorado por la sociedad.

En resumen, a veces parece que los ciudadanos viven con la existencia de dos matemáticas: la escuela, vivida d[oficial]entro de las escuelas, teórica, abstracta y selectiva y la de la vida real que satisface las necesidades emergentes de los ciudadanos. Se percibe que esta "otras matemáticas", rara vez aceptadas por la escuela, se muestra práctica, dinámica y sintonizada con todos los sectores de la sociedad, siendo ampliamente utilizada en las actividades rutinarias de los ciudadanos.

Según D'ambrósio (2001), Ethnomathematics, además de no negar las matemáticas formales, busca llevar a discusión cada forma de Matematizar, analizando argumentativa y reflexivamente cada contexto donde se insertan las matemáticas. Entiende y analiza las diversas formas de Matematizar, siempre que se cumplan los propósitos de cada contexto, de cada grupo de iguales en sus situaciones específicas.

Por lo tanto, trabajar las matemáticas en un enfoque et ${ }^{[10]}$ no, como destate $D^{\prime}$ ambrósio (1993), requiere que el educador aprenda a lidiar con los diferentes conocimientos 
culturalmente construidos y que circulen entre los estudiantes. Propone que la escuela reconsidere su práctica, en armonía con las aspiraciones y necesidades diarias de las personas, rescatando la función social del conocimiento matemático.

En esta dinámica, el educador debe estar abierto a cambios en la forma de actuar y trabajar. Es necesario escuchar las experiencias que los estudiantes traen de casa, de su comunidad, de las relaciones establecidas entre ellos, buscando de este proceso dialogante-interactivo para trabajar el contenido matemático. Y, además de incorporar nuevas metodologías en su práctica, analizando y evaluando constantemente su práctica, mejorando acciones pedagógicas previamente experimentadas. Por último, necesita construir el conocimiento matemático a partir de las experiencias personales de los involucrados en el proceso educativo.

Así, según el profesor Ubiratan D'ambrósio, la práctica de la etnomatemáticaente en el aula, sugiere seguir un camino mucho más amplio de socializar y compartir diferentes conocimientos.

Mediante la práctica de la Etnomathematics, el educador logrará los grandes objetivos de la educación matemática, con distintas perspectivas para diferentes entornos culturales y sistemas de producción. Está justificado insertar al estudiante en el proceso de producción de su comunidad y grupo social y evidencia la diversidad cultural e histórica en diferentes contextos (D'ambrósio, 2008, p, 8).

\section{JUSTIFICACIÓN}

En las escuelas, en un porcentaje considerable, se percibe que el proceso de enseñanza-aprendizaje de las matemáticas sigue siendo inmutable, es decir, el profesor sigue preparando la información, el alumno los recibe pasivamente, transparentemente, en estos casos, Un conformismo acentuado y un determinismo histórico. En la práctica, también existe una metodología desarticulada entre lo que se aprende en la escuela y lo que la sociedad realmente exige a sus ciudadanos. 
Según Santos (2016):

La falta de contextualización en la enseñanza de las matemáticas puede causar el desánimo de la disciplina, resucitando los métodos tradicionales que conceptualizan las matemáticas como una ciencia que preparó todas las cosas, como si fuera un conocimiento y Sobre. (p. 5).

Una enseñanza que lleva al alumno a un aprendizaje mecánico. Además, todavía hay quienes creen que el conocimiento matemático sólo es posible para privilegiados, no accesibles para todos, que según Ubiratan D'ambrósio es comparable al sistema de "casta" aplicado a algunos países.

[...] Algunos educadores matemáticos ven las matemáticas como una forma privilegiada de conocimiento, accesible sólo para algunos especialmente dotados, y cuya enseñanza debe ser estructurada, teniendo en cuenta que sólo ciertas mentes, de alguna manera "especial", pueden Asimilar y apreciar las matemáticas en su plenitu[...]d. (D’AMBRÓSIO, 1996 p.9).

En un intento de minimizar esta situación y llevar motivación y alegría al aula, buscamos a través de esta investigación bibliográfica, tanto una matemática más contextualizada para los estudiantes, como un camino metodológico que proporcione mejores resultados. Una alternativa didáctica que permite una mayor significación de los contenidos de las matemáticas estudiadas. Algo motivador, atractivo y que realmente sirva al alumno de una manera que le permita resolver situacionesproblema de una manera contextualizada, inferir y tomar decisiones frente a su realidad, entendiendo que, incluyendo las matemáticas formales está vivo, está presente, está presente, En nuestro día a día.

Una matemática enseñada en la escuela que está presente en la realidad de la vida de los estudiantes, que busca la interacción entre la escuela y la comunidad, en la posibilidad de ser una herramienta para transformar el entorno social. Un conocimiento cuya construcción y comprensión no sólo es posible para matemáticos, 
científicos, maestros o médicos, sino también para grupos sociales que desarrollan o utilizan en sus actividades laborales habilidades para medir, calcular, dibujar, crear o Juega, de acuerdo a tus intereses y necesidades.

Eso permite a los estudiantes convertirse en temas singularizados y reflexivos, emancipados, confiados e interactivos en la comunidad a través de los conocimientos adquiridos. Giardinetto (1999), en sus escritos reflexiona que el sujeto sólo se convertirá en un ciudadano participante de su sociedad mientras se "apropia de instrumentos culturales" (pág. 73).

Sabemos y estamos de acuerdo en que las matemáticas son una ciencia presente tanto en la vida cotidiana de los ciudadanos como en los centros de investigación. Por lo tanto, considerando este alcance no podemos limitar la enseñanza de esta disciplina a una simple memorización de reglas, técnicas y el conocimiento formal de las definiciones, porque "... La enseñanza no es transferir conocimiento, sino crear posibilidades para su propia producción o su construcción" (FREIRE, 1996, p. 52).

Enseñar matemáticas desde la perspectiva de la etnomatemática se vuelve imposible sin rescatar los conocimientos y valores que los estudiantes aportan de casa. Para que este acto se realice, es indispensable que el maestro escuche a sus alumnos, como es indispensable para el alumno que habla a su maestro y a sus colegas.

Según el educador Paulo Freire,

"La construcción de relaciones dialogantes bajo los fundamentos de la ética universal de los seres humanos, como práctica humana específica implica la conciencia de los seres humanos, para que realmente puedan insertarse en el proceso histórico como sujetos que hacen su Historia propia. " (FREIRE, 1996, p10.)

El educador matemático Paulus Gerdes, en sus diversas publicaciones siempre adopta un discurso que rescata las tradiciones matemáticas de los grupos culturales, 
que antes de la colonización estaban siendo "ocultados" e impuestas por las convenciones eurocéntricas en la sociedad, así como en el espacio escolar.

Así, en esta perspectiva, (GERDES, 2010, p. 142) informa que

La etnomatemática es el área de investigación que estudia las relaciones multifacéticas y las interconexiones entre las ideas matemáticas y otros elementos culturales y constituyentes, como el lenguaje, el arte, la artesanía, la construcción y la educación. Es el área de investigación que estudia la influencia de los factores culturales en la enseñanza y el aprendizaje de las matemáticas.

Según el autor (2010, p. 142-143) "La etnomatemática muestra que las ideas matemáticas existen en todas las culturas humanas, en las experiencias de todos los pueblos, de todos los grupos sociales y culturales, tanto de hombres como de mujeres". Dentro de esta perspectiva cultural, también se evidencia que

[...] Todos los pueblos de todos los tiempos pueden contribuir a estas matemáticas universales. Todos los pueblos tienen derecho a poder aprender y disfrutar de los conocimientos acumulados y de poder contribuir a su enriquecimiento. Aquí se encuentra una dimensión ética y moral de la reflexión de la Etnomatemática. (GERDES, 2010, p.144)

Varios autores ya han informado y por nuestra experiencia profesional también hemos notado que las diferentes formas de Matematizar están tan presentes en la vida cotidiana de los ciudadanos, que muchas personas las utilizan en sus tareas diarias, aunque nunca asistió a una escuela. Este conocimiento, a veces adquirido empíricamente, es de extraordinario valor y riqueza tan significativo que muchos no siempre tienen la sensibilidad para percibirlos. Compartimos con Pompeyo y Monteiro (2001) cuando postulan que,

"El hombre se relaciona con el mundo que lo rodea, lo entiende como la vida mundial, es decir, el mundo en el que las personas están dentro, 
que nos educca a través de influencias que nos llegan a través de nuestras relaciones con él" (pág. 21).

Sin embargo, no se pretende, desde esta perspectiva pedagógica, proponer una nueva matemática, sino reflexionar sobre la posibilidad de identificar, en los diferentes grupos culturales, sus modos de Matematizar, valorando y considerando sus formas de calcular, Conoce, explica y comprende el mundo que los rodea, enriqueciéndolo en esta dinámica matemática escolar. Como informa D'ambrosius (2001), es una matemática que "... Es parte del diario ", del Know-how (pág. 25). De hecho, una propuesta pedagógica que trabaja de manera integrada los conocimientos formales e informales.

Creemos que desde el momento en que pensamos en la Etnomatemática como una alternativa pedagógica que considera las matemáticas en diferentes entornos sociales, culturales e históricos, se incorporan nuevas metodologías en la enseñanza. Se convierte en la escuela matemática un instrumento para explicar y entender el desarrollo y la producción de los conocimientos generados en estos diferentes contextos, motivando a los estudiantes.

Entender el conocimiento que se desarrolla dentro de una comunidad dada es entender la etnomatemática en este entorno, es decir, mirar hacia atrás en las diversas formas de manifestación del conocimiento, no sólo de conocimiento matemático, sino El conocimiento de la vida, de la organización, de la construcción y estructuración de estos conocimientos y haciendo que sean el nacimiento de estas personas.

Sin embargo, muchos educadores de matemáticas, tal vez debido a su educación universitaria centrada en la línea Technicista, muestran dificultades para romper con el viejo paradigma y adoptar una metodología de enseñanza que interconecta las matemáticas escolares con las matemáticas de Vida. Este hecho se convierte en un obstáculo para que el aula se transforme en un espacio de discusión y análisis crítico del conocimiento construido socioculturalmente. Para Pompeyo y Monteiro (2001, p. 19), es necesario buscar formas de superar los conflictos existentes, porque "vivimos 
un momento en que la fragmentación del conocimiento limita la comprensión de la realidad".

Tenemos que estar seguros de que lo que los estudiantes hacen y aprenden en la escuela puede estar más alineado con las acciones y necesidades de los diversos sectores de la actividad humana fuera de los muros de la escuela. El educador simplemente incorpora y trae al aula estos conocimientos construidos socialmente. Compartimos esta investigación con los escritos de D'Ambrosio y Rosa, ya que consideran la Etnomatemática como un programa de investigación en historia y filosofía de las matemáticas, con implicaciones pedagógicas,

Así, este programa de investigación representa una metodología de investigación que busca analizar las prácticas matemáticas locales, ya que busca valorar, difundir y respetar el conocimiento matemático (ideas, nociones, procedimientos, procesos y prácticas) que Se originan en diversos contextos culturales a lo largo de la historia (D'ambrósio y ROSA, 2016, p. 17).

Un trabajo pedagógico centrado en esta perspectiva, valora el ser humano y su conocimiento informal constituido en y por la cultura donde vive, puede ser el comienzo de un cambio en la enseñanza de esta disciplina. Según D'ambrósio (2001), una enseñanza que "revela prácticas incautadas fuera del entorno escolar[pode] contribuyen a una visión crítica de la realidad" (p. 23).

La escuela como institución que representa la continuación de la familia en su contexto más amplio, debe entender que todo el proceso de enseñanza-aprendizaje que parte de las experiencias previas de los ciudadanos, de las formas de matematizar experimentadas por los estudiantes fuera de la Escuela, enriquece el acto educativo. En palabras de Giardinetto (1999) "La vida diaria en sí necesita interferencias del noquotidian" (pág. 7).

En este sentido, dejaría a trabajar las matemáticas oficiales como las únicas matemáticas que merecen atención. Consideraríamos y traeríamos a la discusión las 
experiencias y conocimientos de los estudiantes antes de la fase escolar. La escuela fusionando de forma dialógica todos estos conocimientos transforma el aula en un laboratorio de aprendizaje permanente.

En esta tendencia, uno de los roles de los educadores es estimular el deseo natural de los estudiantes de investigar las diferentes matemáticas utilizadas en los diversos grupos culturales. Al entender los procesos de Matematizar de cada cultura, comparan estos conocimientos con conocimientos formales, systate y validados por la escuela, teniendo la oportunidad de organizar su propio pensamiento, comprendiendo a través de esta reflexión la importancia cultural y social de todos los conocimientos matemáticos que circulan en el mundo de la vida. En palabras de D'ambrósio (2001), podría entenderse mejor que "el comportamiento de cada individuo, asociado con su conocimiento, se modifica por la presencia del otro" (pág. 32).

Según D'ambrósio (1993), enseñar matemáticas en esta concepción significa respetar lo que el estudiante ya sabe, consiste en reconocer el conocimiento históricamente construido en las culturas, establecer momentos dialogados de cuestiones críticas que renuevan y El conocimiento previo de todos los participantes en el proceso de aprendizaje.

Para D'ambrósio (2001) Respeta y valora cada una de las matemáticas existentes, y "... Es la comunicación la que permite definir estrategias de acción común "(p. 58). La escuela, por reflexión dialéct ${ }^{[11] i c a, ~ e x p l i c a ~ l o s ~ m o d o s ~ d e ~ p r o d u c c i o ́ n ~ d e ~ s i g n i f i c a d o s ~}$ matemáticos producidos fuera de su contexto específico. En este enfoque es como si la escuela matemática se uniera a las formas de matematizar del mundo circundante, en lugar de reemplazarlo. Mientras que las matemáticas escolares ignoran o no discuten esta diversidad de conocimientos, producida según la cultura de cada pueblo, la etnomatemáticatigia valora y trae al debate estas diferencias, reconociendo sus modelos matemáticos y los conocimientos vinculados a ellos.

Según D'ambrósio (2001), mientras que las matemáticas tradicionales y su enseñanza buscan universalizar los conceptos, haciéndolos cada vez más abstractos y 
generalizados, la Etnomatemática busca reorganizarlos, haciéndolos más específicos y aplicables a Contexto de la realidad vivida. Propone, en resumen, una forma más coherente de caminar. Busca llevar las matemáticas de los I[da escola]ibros a la vida y a la vida a la escuela, reconociendo que ambos tienen una historia e importancia para entender la realidad en todas sus dimensiones.

La idea básica de esta tendencia a enseñar matemáticas, según D'ambrósio (2001), es considerar las experiencias de estudiantes, familiares, grupos de iguales, diferentes culturas y todas las formas de auto-matematizar de cada pueblo. Son conocimientos producidos por la necesidad práctica de resolver problemas específicos de fairers, pequeños agricultores, albañiles, carpinteros, grupos indígenas, colonos, etc. Son diferentes conocimientos producidos por diferentes grupos para situaciones problemáticas elucideales.

Para Pompeyo y Monteiro (2001), una práctica pedagógica que al mismo tiempo se opone a la fragmentación del conocimiento, se caracteriza por situar el conocimiento histórico cultural y oportunizar a los hombres para el rescate de su capacidad dialogante reflexiva crítica. Entiende, según los autores, al ser humano en su totalidad y al conocimiento en toda su complejidad.

En palabras de Pompeyo y Monteiro (2001), una enseñanza que sitúa "el hombre y no el capital como valor central de las relaciones sociales" (P. 26). Según D'ambrósio (2001), un trabajo centrado en esta perspectiva requiere que los educadores tengan una buena dosis de humanismo, porque "el estudiante es más importante que los programas y el contenido" (pág. 86).

Las matemáticas trabajadas en un enfoque etno trae a las experiencias en el aula que requieren comunicación y diálogo argumentativo. Esta postura didáctica, a su vez, lleva a los involucrados en el proceso educativo a un crecimiento personal y colectivo, hecho posible por la acción reflexiva permanente de ambas, y de ambas prácticas.

Juntos, profesores y estudiantes enseñan y aprenden simultáneamente, conocen el mundo en el que viven críticamente y construyen relaciones de respeto mutuo, justicia, 
constituyendo un verdadero clima de disciplina, mediante relaciones dialogantes, haciendo del aula un desafío Interesante y desafiante para todos los involucrados. "EI que enseña aprende cuando enseña y aprende enseña en el aprendizaje" (FREIRE, 1996, p. 38).

Según Giardinetto (1999), "El maestro puede y debe utilizar el conocimiento diario como punto de apoyo al proceso de enseñanza-aprendizaje" (pág. 68), llevando al estudiante al campo de la argumentación, desarrollando el hábito de la lectura crítica, la investigación, Cuestionamiento, de creatividad, indispensable para la formación ciudadana.

Al lanzar esta mirada en su práctica, se valora el conocimiento previo de los estudiantes y se forman otros, siempre a partir de los conocidos. Lo importante es el establecimiento de lazos de conocimiento de la comunidad con el conocimiento de la escuela y el conocimiento de la escuela con el conocimiento de la comunidad. A través de este establecimiento y estas relaciones es posible atribuir significado a ambos conocimientos. Para D'ambrósio (2001), en este contexto, el conocimiento presenta un carácter dinámico, siempre abierto a nuevos enfoques.

Para Freire (1996) El papel del maestro está vinculado a la concepción de que la enseñanza no es transferir conocimiento, sino crear condiciones para su construcción. Significa reconocer que juntos, los estudiantes y los maestros aprenden en el salón de clases, ya que todos aportan muchos conocimientos de las experiencias escolares y no escolares que han experimentado durante su existencia.

Creemos que la Etnomatemática puede ayudar a cada educador en esta posibilidad pedagógica de reemplazar las acciones rutinarias de "dar clases listas" por una postura que (re) oriente el aprendizaje del estudiante en la construcción de sus propios conocimientos, fruto de Interacción entre sujetos en este proceso dialogante. Aun porque la sociedad del conocimiento en la que vivimos, exige hoy una postura holística en la formación de sus ciudadanos, que son capaces de interactuar con el dinamismo 
en todos los contextos, construir nuevos significados para las matemáticas, explorar Diferentes formas de situaciones-problema de diversas actividades humanas.

\section{GOL}

Contribuir desde la reflexión al educador de matemáticas para adoptar una postura crítica de investigación en relación con la enseñanza de las matemáticas, desde la perspectiva de iniciar su actividad docente a través del establecimiento de vínculos entre las matemáticas diarias y Matemáticas escolares.

\section{METODOLOGÍA}

Las inspiraciones teóricas de este artículo se deben a lecturas y reflexiones sobre matemáticas Educación, Etnomatemática y la perspectiva intercultural de la enseñanza. Los escritos buscan reflexionar sobre el enfoque etnomatemático en la perspectiva de que una enseñanza basada en los conocimientos previos de los estudiantes, valorando su cultura y sus modos de Matematizar, será un factor de conocimiento agregativo, dando un carácter más humano Por disciplina.

\section{CONTRIBUCIONES PREVISTAS}

Que se perciba, según D'ambrósio (2001), la importancia de la etnomatemáticaente en la formación del alumno, ya que su sesgo cultural y antropológico apunta al camino que lleva al respeto de las diferencias, la comprensión y la inclusión, tan necesarios para nuestra realidad Social.

Que la enseñanza de las matemáticas en el aula, desde esta perspectiva pedagógica, permite un diálogo eficaz entre los conocimientos matemáticos producidos en diferentes contextos y que los profesores reflexionan más sobre la diversidad cultural de la disciplina y su Importancia para la educación de Systate. 
Que a partir de esta reflexión la práctica pedagógica en el aula puede reorientarse al desarrollo de la plena ciudadanía de los alumnos. Que, según Pinheiro y Rosa (2016), el:

[...] Los profesores de matemáticas se sumergen en la dinámica cultural de los estudiantes y utilizan estrategias de enseñanza y aprendizaje que valoran la dimensión cultural en el aula, para que se pueda desarrollar una educación matemática inclusiva que pueda desarrollarse eficazmente Contribuir a la transformación social (pág. 79).

Que este esfuerzo puede contribuir a nuevos elementos para enseñar la disciplina de las matemáticas, permitiendo en el aula estudiar cada vez más matemáticas y realidad, como formas de conocer e interpretar cada contexto, en un proceso en el que el maestro estará Constantemente (re) producir, (re) construir, (re) significado de conocimiento y conocimiento.

También esperamos que los lectores perciban la posibilidad de insertar en la enseñanza de las matemáticas una mirada diferenciada a la realidad y a las matemáticas en sí y que este aspecto se corroe con el aprendizaje matemático de la educación para llevarte a participar en Debates sobre matemáticas dentro y fuera del aula.

Queremos que el aula, basada en la metodología de enseñanza, comience a incorporar/analizar en el proceso pedagógico, situaciones problemáticas cuya resolución considere tanto las matemáticas formales como las matemáticas informales. Que la disciplina sea vista como una actividad humana, motivando aún más el interés de los estudiantes.

Destacamos que no se trata de sustituir las matemáticas por otra, sino de respetar los diferentes modos de Matematizar en diferentes contextos, dentro de los fines a los que se proponen. Por lo tanto, creemos que este enfoque educativo puede referirnos a una educación contextualizada, enriquecida, motivando a los participantes a ser parte del proceso. 
En palabras de RAM uno,

[...] Enseñar matemáticas en esta concepción permitirá al alumno vincular los conceptos que trabajan en clase con su experiencia diaria, de acuerdo con su entorno natural, social y cultural. No se trata de rechazar las matemáticas académicas, sino de incorporar valores que se experimentan en experiencias grupales, teniendo en cuenta los lazos histórico-culturales (CARNEIRO, 2012, p. 3).

Por último, pretende contribuir a la Constitución del investigador profesor de su práctica, despertándole a aprender de forma continua para ser profesor, de acuerdo con las necesidades educativas actuales, transformando el aula en un entorno de aprendizaje Intercambio democrático de conocimiento, rescatando el conocimiento histórico-socialmente construido de la humanidad.

\section{REFERENCIAS BIBLIOGRÁFICAS}

CARNEIRO, K. T. A. Cultura Surda na aprendizagem matemática da sala de recurso do Instituto Felipe Smaldone: uma abordagem etnomatemática. Anais do 4ํㅡㄹ Congresso Brasileiro de Etnomatemática. Belém, PA: ICEm4, 2012.

D'AMBRÓSIO, Ubiratan. O programa Etnomatemático: Uma síntese. Acta Scientía, v.10, n.1, Jan/jun.2008.

. Etnomatemática - elo entre as tradições e a modernidade. Coleção Tendências em Educação Matemática, 1. Belo Horizonte: Autêntica, 2001, 112p.

Da realidade a ação: Reflexões sobre a educação e matemática. Campinas: Unicamp, 1996.

. Etnomatemática: Arte ou técnica de explicar e conhecer. Editora Ática, Série Fundamentos, 2. edição, São Paulo, 1993. 
D'AMBROSIO, U.; ROSA, M. Um diálogo com Ubiratan D'Ambrosio: uma conversa brasileira sobre etnomatemática. In BANDEIRA, F. A.; GONÇALVES, P. G. F. (Orgs.). Etnomatemáticas pelo Brasil: aspectos teóricos, ticas de matema e práticas escolares. Curitiba, PR: Editora CRV. 2016. pp. 13-37.

FREIRE, Paulo. Pedagogia da autonomia: Saberes necessário a prática educativa. São Paulo: Paz e Terra, 1996.

GERDES, Paulus. Geometria dos Trançados Bora na Amazônia Peruana, Livraria da Física, São Paulo, 2010, 190 p. São Paulo: Editora Livraria da Física, 2011, 190p.

GIARDINETTO, José Roberto Boettger. Matemática escolar e matemática da vida cotidiana. Coleção polêmicas do nosso tempo, autores associados, Campinas - São Paulo, 1999, 128p.

MONTEIRO, A.; Pombeu, G. Jr. A matemática e os temas transversais. Editora Moderna, São Paulo, 2001, 160p.

PINHEIRO, R. C.; ROSA, M. Uma perspectiva etnomatemática para o processo de ensino e aprendizagem de alunos Surdos. RPEM, v. 5, n. 9, p. 56-83, 2016.

ROSA NETO, R. Didática da matemática, São Paulo: Ática, 2002.

SANTOS, Lijecson Souza dos. Contextualização Matemática Em Situação De Ensino E Aprendizagem No Eja. Disponível em: https://editorarealize.com.br/revistas/cintedi/trabalhos/TRABALHO_EV060_MD1_SA 18_ID 492_23102016194610.pdf. 2016.

8. En este trabajo de investigación, utilizado para designar referencias metodológicas que pueden desarrollarse desde la perspectiva de la investigación de las concepciones, tradiciones y prácticas matemáticas de un grupo social, a través de la cual el grupo interpreta y codifica su Conocimiento. 
9. Ubiratan D'ambrósio, profesor, educador matemático e investigador de la Universidad Estatal Paulista (UNESP). Campus Río Claro. Instituto de Geociencias y Ciencias Exactas (IGCE). Es profesor emérito de la Universidad Estatal de Campinas/UNICAMP. Ha publicado estudios en: Historia y Filosofía de las Matemáticas, Historia y Filosofía de las Ciencias, Etnomatemática, Etnociencia, Educación Matemática y estudios transdisciplinarios.

10. El enfoque etno se refiere a varias perspectivas en circulación con respecto a esta área, que no es, hasta la fecha, teóricamente cristalizada. Los educadores e investigadores involucrados en este tema decidieron dar tiempo para que las exploraciones avanzaran y así contribuir a una posible elaboración teórica de etnomatemática. Es posible que este desarrollo incluya ampliamente las diversas perspectivas en circulación.

11. La dialéctica en esta reflexión, expresa el arte de buscar el diálogo, a través de la argumentación y contrarrestar la expansión de las ideas y conceptos involucrados en la discusión. En el análisis de los hechos y situaciones-problema que permiten el desarrollo del pensamiento, el maestro y el estudiante tienen la misma participación dialogante, uno siempre escuchando al otro, (re) construyendo juntos sus aprendizajes.

Enviado: Junio de 2019.

Aprobado: Julio de 2019. 